\title{
Profile of the Institute for Rehabilitation and Research
}

\author{
WH Donovan \\ Professor and Chairman, Department of Physical Medicine and Rehabilitation, University of Texas, Houston Medical \\ School, The Institute for Rehabilitation and Research (TIRR), 1333 Moursund, Houston, Texas 77030-3405, USA
}

Keywords: spinal cord injury unit; neuro-intensive care; initial and subsequent management; rehabilitation; teaching; research

\begin{abstract}
History
The Institute for Rehabilitation and Research (TIRR) a private, not for profit, rehabilitation hospital, was founded in 1959 in Houston's Texas Medical Center by William A Spencer, MD. Dr Spencer articulated a rehabilitation philosophy of maximizing independence and quality of life that continues to guide the development of all the treatment programs. This guiding philosophy includes providing appropriate medical intervention, helping the patient establish and accomplish realistic goals and objectives, and supporting the patient to maintain personal integrity and family and social ties. TIRR is an internationallyknown hospital which specializes in medical care, education, and research in the field of catastrophic injury. Recently rebuilt, it has been recognized every year for the past seven (7) years in a nationwide survey of physicians by US News \& World Report magazine as one of the best rehabilitation facilities in America (Figure 1).

From a strong foundation in the rehabilitation of patients with poliomyelitis, additional programs in rehabilitation evolved utilizing the experience and expertise for the treatment of people sustaining catastrophic injuries. One of these programs is the Spinal Cord Injury (SCI) Program.
\end{abstract}

\section{SCI program}

More than 3000 SCI patients have been through the TIRR Spinal Cord Injury Program since its inception in 1962. TIRR maintains close liaison with the two adjacent Level 1 trauma centers within the Texas Medical Center: Hermann Hospital and Ben Taub General Hospital. These two trauma centers capture virtually $100 \%$ of the spinal cord injury patients within a 150 mile radius. Patients are brought to these centers by surface and helicopter transport. Outside this radius TIRR also receives most of the SCI patients from cities located in a catchment area of 200 to 350 miles beyond the central radius. In addition,

Correspondence: WH Donovan
TIRR receives certain referrals from all areas of the country. Within the catchment area, acute care is administered via a sophisticated EMS system, at the Level 1 trauma center's emergency room (ER) and in specially equipped neuro-intensive care units (NICU's). All patients are seen immediately by a neurosurgeon upon arrival in the ER and all who arrive within $8 \mathrm{~h}$ of the injury received methylprednisolone according to published protocols. If patients require surgical stabilization, this is done at the trauma center by a neurosurgeon or by a neurosurgeon and orthopedist working together prior to transfer. When the patient is stabilized to where he/ she no longer requires intensive care, transfer to the rehabilitiation setting at TIRR is immediately carried out. All patients regardless of their ability to pay who are treated at the trauma centers are transferred to TIRR for comprehensive rehabilitation. Most arrive at TIRR within the first two weeks of injury but whenever they no longer require intensive care. In the NICU, the neurosurgeon is the primary director of care. In the rehabilitation setting, the physiatrist is the primary physician. Consultants may include a pulmonologist, urologist or other specialists depending on the presence of co-morbidity.

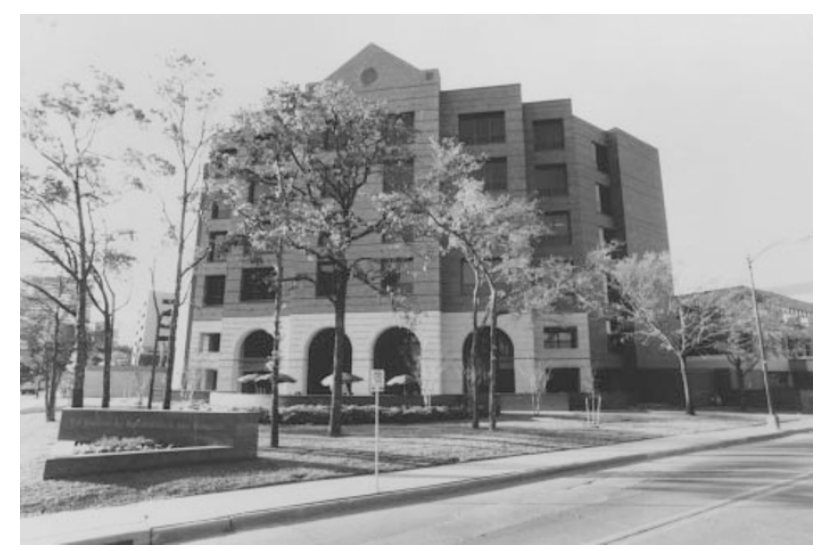

Figure 1 The Institute for Rehabilitation and Research (TIRR) in the Texas Medical Center, Houston, TX, USA 
Before admission, families are encouraged to tour the facility and are given a detailed manual on spinal cord injury at the outset. Results of the patient's initial examination and plan of care are discussed with both patient and family at great length. Medical management of the patient's program is coordinated and prioritized by the patient's own spinal cord physician (physiatrist) who determines the specific strategy to maximize function as quickly as possible. The rehabilitation team also includes nurses who are skilled in medical/surgical care and rehabilitation nursing, physical and occupational therapists, speech therapists, registered dieticians, therapeutic recreation specialists, and social workers. Other disciplines, such as respiratory therapy and psychology are available according to patient needs. All work closely with the physician to meet discharge goals. Team members confer with one another daily on the patient's status, and meet weekly at the team conference with the physician and patient (and family) to evaluate progress and to ensure the continued effectiveness of the patient's treatment plan. At this time, each long and short term goal is reviewed and updated, progress is documented, and the family and patient may ask any questions they like.

To help the patient and family understand the effects of spinal cord injury on bodily function, a series of educational classes is followed by individualized instruction. Education is an integral part of the patient's treatment. Each member of the team assesses information and education needs (Figure 2).

Throughout the rehabilitation hospitalization, the internal case manager (usually a Registered Nurse) remains the family's chief point of contact. This individual keeps abreast of all the activities of the rehabilitation team, acts as the key individual to communicate progress to the funding agency and is available to keep the family fully informed.

The ultimate goal of this SCI Program is to enable patients to achieve the maximum possible level of independence, productivity and health. To that end,

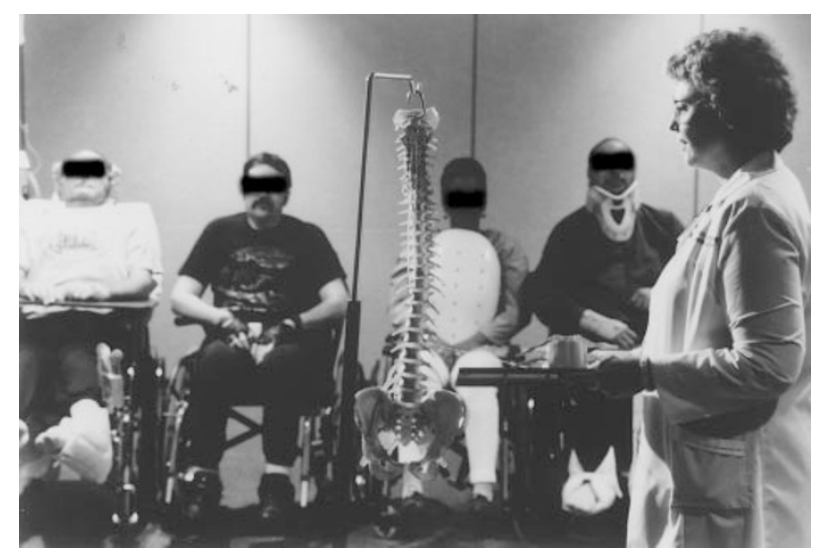

Figure 2 Patients attending 'RAPS' - an education series attended by all SCI patients the activities of the program are designed to maintain overall health, prevent complications but treat them competently if they should arise, improve the patient's strength, endurance and function to the point that further progress will be possible after discharge, prescribe the appropriate equipment, prepare the patient and family to follow a home program successfully, and provide a means for life long follow-up (Figure 3).

The range of services available generally includes: medical management by a primary SCI physician who consults appropriate medical/surgical sub-specialists when needed, occupational therapy, physical therapy, rehabilitation nursing, respiratory therapy, social services, and therapeutic recreation (Figure 4).

If needed, patients are also provided with such services as intermittent catheterization, advanced diagnostic testing, audiology, chaplaincy, driver education, dietetics, orthotics, advanced technology/ bioengineering, psychology/neuropsychology, special education, speech-language pathology, and vocational rehabilitation.

Table 1 shows the distribution of patients by level of injury who were discharged from TIRR in 1996.

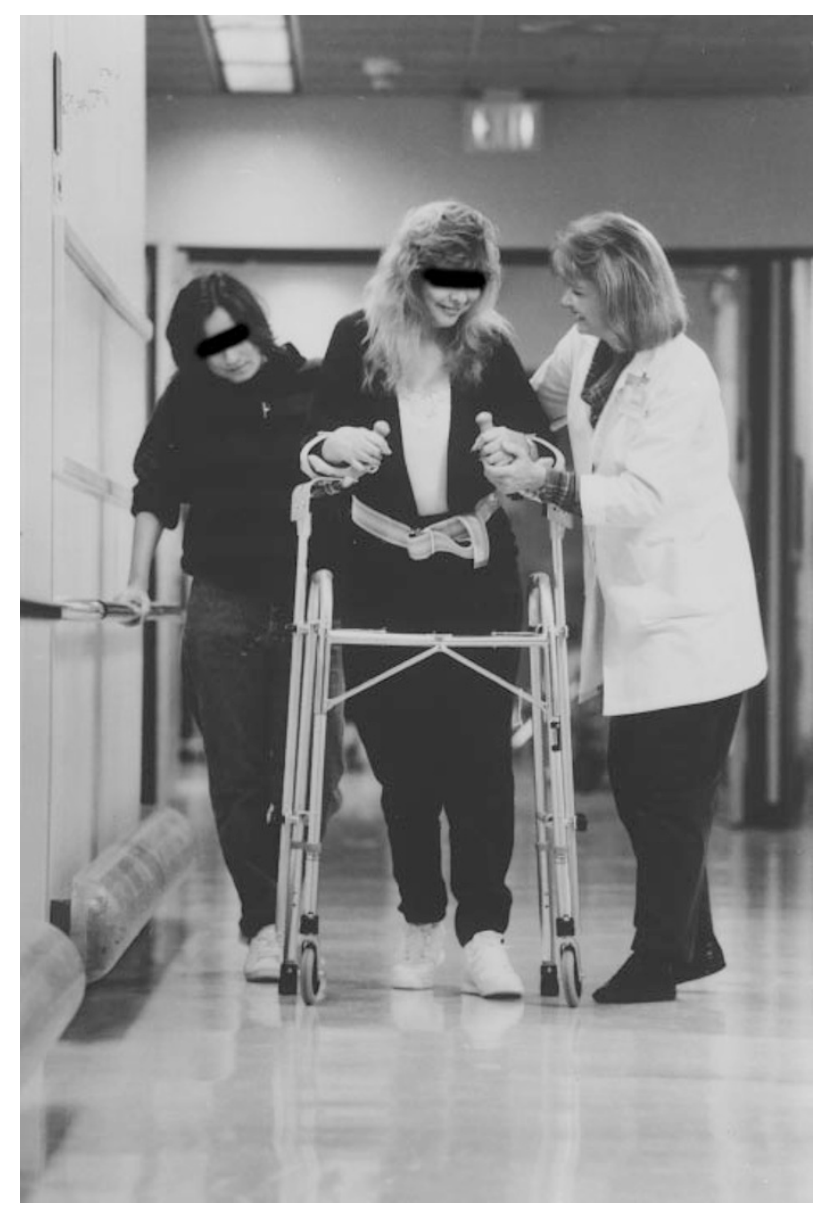

Figure 3 An incomplete tetraplegic patient re-learning to walk 


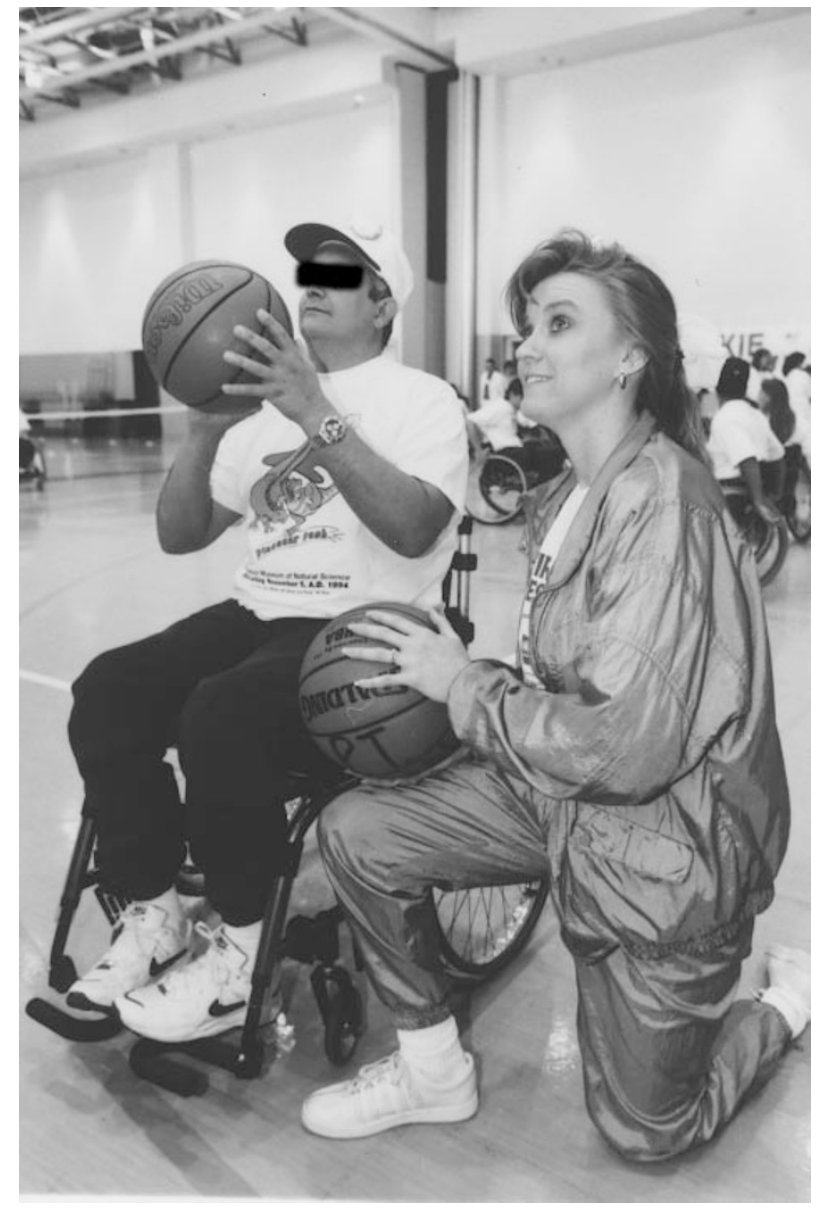

Figure 4 An L1 paraplegic getting some coaching from his therapist on his 3-point shot

Table 1 Breakdown of functional level of patients discharged from TIRR SCI program in 1996

\begin{tabular}{lrc}
\hline Functional level & $N$ & Statistics \\
\hline C1-C4 & 19 & Percent \\
C5-C8 & 35 & 16.4 \\
T1-S5 & 56 & 30.2 \\
Missing & 6 & 48.3 \\
Total & 116 & 5.2 \\
\hline
\end{tabular}

Some of the special services available to SCI patients at TIRR are as follows.

\section{Baclofen pump}

TIRR was one of the first hospitals in the country to offer a surgically implanted, computerized infusion pump that delivers continuous doses of baclofen, a spasticity-reducing drug into the spinal fluid, for those with spinal cord injury. The continuous infusion of a small amount of baclofen intrathecally relaxes the spasticity and provides constant relief for spinal cord injury patients as well as those with multiple sclerosis, head injury or cerebral palsy.

\section{Seating/mobility}

The Seating and Mobility Clinic at TIRR meets the needs of individuals who require specialized wheelchair and seating equipment. The clinical staff evaluate the client's abilities, measure the pressures under the patient's seating surfaces, make recommendations about the seating systems that best relieve the pressure, fit the systems once they are approved, and provide training as necessary. Staff also advise and assist with the choice of a wheelchair (or scooter), and also provide instruction as to the appropriate methods to relieve tissue pressure.

Equipment shown and tested in the clinic includes: manual wheelchairs, power wheelchairs, switch and special controls accessories, seat and back cushions, hand supports, linear seating systems, and modular seating systems.

\section{High tetraplegia}

Some spinal cord injured patients seen in the SCI program require a mechanical ventilator to sustain their breathing either temporarily or permanently. These individuals come to TIRR because the hospital is known for its expertise and experience in the management of ventilator-dependent spinal cord patients, as well as those implanted with electrophrenic simulators used in place of the mechanical ventilator.

\section{Adaptive driving program}

TIRR provides an Adaptive Driver Program for persons with physical limitations due to spinal cord injury, as well as traumatic brain injury, stroke, amputation and a wide range of neuromuscular and orthopedic conditions.

The evaluation process consists of a clinical evaluation followed by a behind-the-wheel evaluation to determine the individual's capabilities. On-the-road teaching is done in either a fully-equipped van or car. Following the teaching program, prescriptions are generated for any adaptive equipment required.

\section{TIRR sports}

TIRR Sports is a community service organization committed to enhancing the lives of individuals with physical disabilities through reaction and sports. The program provides people with mobility limitations a wide variety of competitive sports, recreational and exercise activities, and seminars. Sports in the program include swimming, wheelchair basketball, wheelchair handball, bowling, and track and field events. New activities, based on the interest and suggestions of participants, are introduced periodically. TIRR believes 
in the importance of making sports and exercise available to people with mobility limitations, for a healthier, happier and more productive lifestyle (Figure 5).

\section{Education}

TIRR has a long-standing commitment to educating health professionals, patients and families, and the public about rehabilitation. TIRR conducts educational conferences that bring together patients and families, rehabilitation professionals, community providers of services, payors and policy makers. These conferences are used to report the results of TIRR's and other investigators' pertinent research projects and to provide practical information and hands-on instruction. Topics discussed at these conferences include sexuality and disability, physical therapeutic treatment of the patient's spinal cord injury, e.g., functional electrical stimulation, the latest developments in research on cure in spinal cord injury, special issues of women with disabilities, therapeutic interventions e.g., botulinum toxin, serial casting, and advances in assistive technology.

The hospital develops educational materials and provides instruction through individual and group teaching sessions, videotapes, manuals and other written materials to patients and families about disability and the opportunities and problems they may have in everyday life.

TIRR provides special programs designed to educate the community about the Americans with Disabilities Act (ADA) and to advocate for the rights of people with disabilities. As part of the national Think First program, TIRR speakers promote safety measures that reduce the risk of injuries through presentations to school children, parents, and business and volunteer organizations.

TIRR also has contracts with the two medical schools, the University of Texas Medical School Houston and Baylor College of Medicine who provide the faculty and residents who direct the patient's care.

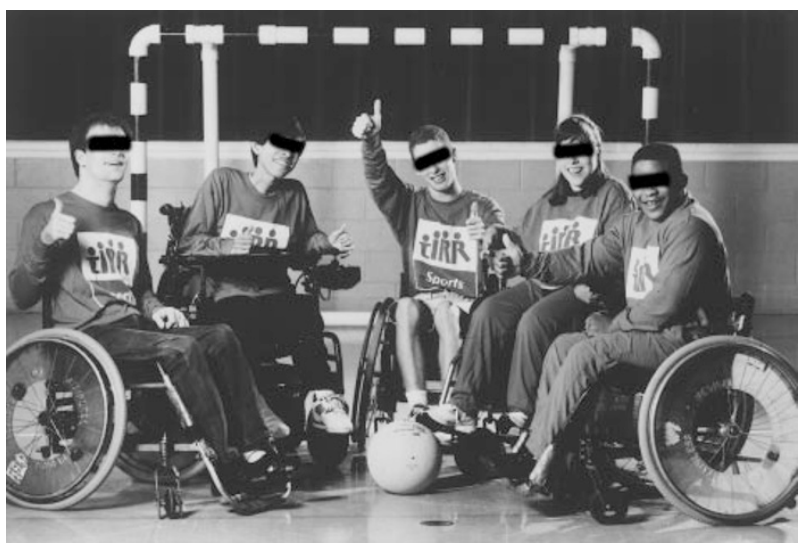

Figure 5 A few players and supporters gather after a victory
Six residents continually rotate through TIRR every two months drawn from the 45 residents in the combined physical medicine and rehabilitiation (PM\&R) residency program. Other allied health students rotate in their respective departments, e.g., PT, OT, speech, pharmacy, dietary at specified intervals as well. TIRR has currently 15 teaching videos and patient care manuals in English and Spanish that are used in teaching and are for sale.

\section{Research}

TIRR is an internationally recognized center for research in medical rehabilitation. TIRR's research activities are aimed at understanding the causes and consequences of physical disability and associated impairments and at developing improved treatment procedures and assistive technology for minimizing disability.

Research initiatives include service demonstrations which develop and evaluate new techniques and approaches designed to enable people with disabilities to be fully integrated into community life. Among these demonstrations are federally-sponsored Model Systems for both spinal cord injury and brain injury people. These Model System centers improve the availability and quality of services for people recovering from such occurrences.

TIRR support for research is augmented by grants from numerous sources including The National Institute on Disability and Rehabilitation Research (NIDRR), the Rehabilitation Services Administration of the U.S. Department of Education, the National Center for Medical Rehabilitation Research (NCMRR) at the National Institutes of Health (NIH), the Robert Wood Johnson Foundation, and others. Current ongoing investigations include: The Effect of 4-Aminopyridine on Long Term SCI, The Effect of Bacterial Inhibition on Urinary Tract Infections, New Applications for Telemedicine, Medical and Social Problems Unique to SCI Women, Methods to Relieve Tissue Pressure and many others. The hospital's research commitment keeps it at the forefront of advances in rehabilitation. 4-Aminopyridine (4AP) is a potassium channel blocker that has been reported to enhance conduction in partly injured nerve fibers. A double blind, cross over study has just been completed and the data are being analyzed. The 4AP was injected intravenously and its effect on the neurologic examination and neurophysiologic studies was measured. We hope to publish the results in Spinal Cord in the near future.

Despite reductions in reimbursement for hospital and outpatient treatment and a lessening of government sponsored research grants, TIRR remains committed to continue to serve as a model for health care delivery to patients with catastrophic injuries including spinal cord injury and maintain its leadership role in education and research as we enter the next millennium. 